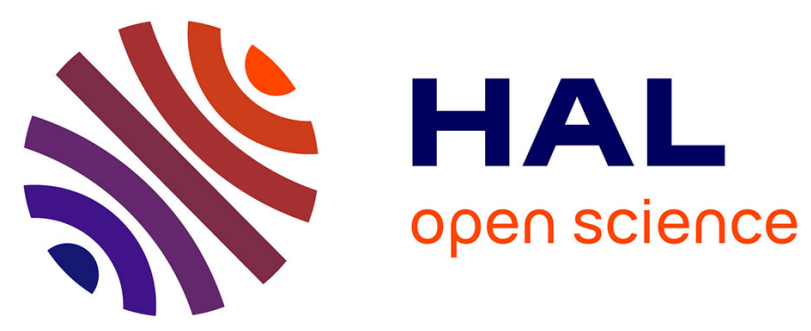

\title{
Path tracking of a four-wheel steering mobile robot: A robust off-road parallel steering strategy
}

\author{
M. Deremetz, R. Lenain, A. Couvent, Christian Cariou, B. Thuilot
}

\section{To cite this version:}

M. Deremetz, R. Lenain, A. Couvent, Christian Cariou, B. Thuilot. Path tracking of a four-wheel steering mobile robot: A robust off-road parallel steering strategy. ECMR The European Conference on Mobile Robotics, Sep 2017, Paris, France. hal-02607237

\section{HAL Id: hal-02607237 \\ https://hal.inrae.fr/hal-02607237}

Submitted on 16 May 2020

HAL is a multi-disciplinary open access archive for the deposit and dissemination of scientific research documents, whether they are published or not. The documents may come from teaching and research institutions in France or abroad, or from public or private research centers.
L'archive ouverte pluridisciplinaire HAL, est destinée au dépôt et à la diffusion de documents scientifiques de niveau recherche, publiés ou non, émanant des établissements d'enseignement et de recherche français ou étrangers, des laboratoires publics ou privés. 


\title{
Path tracking of a four-wheel steering mobile robot: A robust off-road parallel steering strategy
}

\author{
Mathieu Deremetz ${ }^{1}$, Roland Lenain ${ }^{1}$, Adrian Couvent ${ }^{1}$, Christophe Cariou $^{1}$ and Benoit Thuilot ${ }^{2,3}$
}

\begin{abstract}
In this paper, the problem associated with accurate control of a four-wheel steering mobile robot following a path, while keeping different desired absolute orientations and ensuring different desired lateral deviations, is addressed thanks to a backstepping control strategy. In particular, the control of each steering angle is investigated through a new parallel steering approach based on an extended kinematic model of a bicycle-model robot assuming that the two front steering angles are equal and likewise for the two rear ones. Two control laws are then proposed to ensure a suitable path following according to orientation and position conditions. In order to balance the lateral effects, notably the sideslip angles, an observer has been used to estimate the sliding. This estimation permits to feed the proposed control laws appropriately, enabling an accurate path tracking and orientation keeping along the trajectory. This new point of view permits to achieve difficult manoeuvres in narrow environments such as a parallel parking or sharp turns. Previous approaches have focused on the control of four-wheel steering mobile robots with respect to the trajectory but do not combine path following with independent heading angle control and slippery conditions.
\end{abstract}

\section{INTRODUCTION}

Initiated by W. Grey Walter thanks to Elmer and Elsie robots in [5], mobile robotics constitutes today a major economic and scientific topic. One of the best examples is the progress achieved on autonomous vehicles, notably cars [11] which are now close to the market. The transport sector is not the only field that benefits from mobile robotics. Indeed, since the beginning of the twenty-first century, research has focused its interest on the automation of high-speed vehicles in off-road context. Thus, sectors such as agriculture [2] or even defence and space [15] are considering autonomous ground vehicles in order to achieve painful tasks, submitted to very harsh and variable conditions.

Over the last few years, it can be noticed that mobile robotics developments are not only linked to automated manned vehicles but also to totally autonomous adaptable robots [12] that have more and non-standard mobility to be piloted. Concerning guidance, firstly based on the frontsteering model used for cars, these mobility levels have indeed evolved through adding a rear steering axle, skidsteering configuration or using holonomic wheels. Another option, investigated in this paper, is the use of four independent steering wheels. Because of its high mobility, this

1 Irstea, Technologies and Information Support System Research Unit, 9 avenue Blaise Pascal, CS 20085, 63178 Aubière, France firstname. lastnamedirstea.fr

2 Clermont Université, Université Blaise Pascal, Institut Pascal, BP 10448, 63000 Clermont-Ferrand, France firstname. lastname@univ-bpclermont. fr

${ }^{3}$ CNRS, UMR 6602, Institut Pascal, 63178 Aubière, France configuration, compared to the latest, is well appropriate for performing tricky moves on road or in off-road contexts. Such a robot, equipped with four steering wheels, is then appropriate to achieved path tracking requiring suitable control of both heading and orientation.

Several approaches have been proposed in the framework of path following. Firstly developed for front-steering [13] or skid-steering [4], [8] mobile robots, classical approaches are designed using pure rolling without sliding assumption, as developed by [13] or [16]. Nevertheless, if the robot has to follow a trajectory at medium speed (beyond $2-3 \mathrm{~m} / \mathrm{s}$ ) the accuracy of the tracking may be seriously damaged, especially in off-road context. Then, sliding has to be taken into account to preserve the accuracy of the path tracking. This approach is well demonstrated for front-steering mobile robots in [9]. However, this approach only ensures the control of the lateral deviation of the robot w.r.t. the trajectory. Indeed, a crab angle between the orientation of the robot and the tangent to the path appears when the robot moves on a slope. To avoid this phenomena another control strategy has been proposed in [3] for four-wheel steering mobile robots in order to take advantage of both front and rear steering actuations to control both the lateral deviation and the angular deviation of the robot w.r.t. the trajectory during the path tracking. This latter approach is notably designed for autonomous vehicles with limited steering angles because of Ackermann steering geometry. Moreover, the orientation of the robot is controlled w.r.t. the trajectory. Another method in [17] proposed a parallel steering with equal front and rear steering angles but did not suggest a robust method to avoid the skid and the deviation from the desired path when grip conditions are poor.

With the raise of unmanned adaptable robots equipped with four independent steering wheels having a huge range of movement (beyond $\frac{\pi}{2}$ or even $\pi$ radians) such as the Thorvald platform [6] or the agricultural robot proposed in [7], new kinds of movement during path tracking can be achieved. Indeed, if there is no passenger, the angular error of the robot w.r.t. the trajectory does not have to reach zero anymore. From this point of view, one can then imagine controlling a robot with a desired absolute orientation during the path tracking, which permits to substantially reduce the manoeuverability space.

In this paper, the autonomous following of a path with a desired absolute angle by a four-wheel steering mobile robot is investigated. More precisely, a backstepping approach has been developed in order to compute the front steering angles and the rear steering angles based on an extended kinematic 
model. To compensate for the effects of the grip conditions, an absolute observer, based on prior work [10], estimates the sideslip angles and feeds the proposed control laws in terms of sliding.

This paper is decomposed as follows. First, the extended kinematic model is recalled. The second part details the observer used to estimate the grip conditions of the robot wheels, while the third section describes the proposed control laws based on a backstepping strategy. Finally, simulations are conducted to demonstrate the results and the efficiency of the proposed approach and are illustrated through a few agricultural contexts.

\section{MODELING}

\section{A. Mobile robot modeling}

The objective of the proposed application is to allow for a four-wheel steering mobile robot to follow a previously defined trajectory (i.e., computed, previously learned or coming from a structure to follow). This trajectory may include turns and ground variations which may lead to sliding and then create grip condition modifications. To ensure accurate tracking by using an observer and a control algorithm, it is appropriate to use models that take into account the kinematics of the robot.

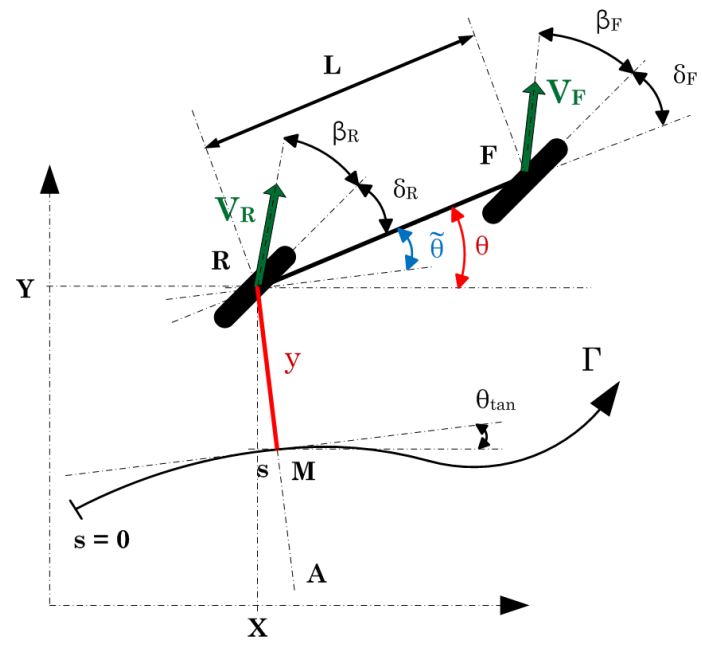

Fig. 1: Extended kinematic model of the robot with respect to the reference trajectory $\Gamma$ and in the absolute frame defined by the $X$ and $Y$ coordinates.

In this paper, a four-wheel steered and drive robot is considered. As is commonly assumed, the robot is viewed as a bicycle, with an equivalent front steering angle $\delta_{F}$, an equivalent rear steering angle $\delta_{R}$ and a wheelbase L (Fig. 1). The robot's speed is defined thanks to the speed of the front axle $v_{F}$ and the speed of the rear axle $v_{R}$. The bicycle model is commonly used in the field of mobile robotics, but it is generally supposed that the rolling without sliding condition is satisfied. In an off-road context or at high speed, however, this assumption cannot be satisfied and leads to a lateral deviation of the robot during the tracking when control based on such a model is implemented, as pointed out in [1]. To overcome this phenomenon and take into account the non-ideal grip conditions, two additional variables, $\beta_{F}$ and $\beta_{R}$, are added. These variables, called sideslip angles, denote the front and rear angles, respectively, between the tire orientation and the current speed vector orientation at the contact points $F$ and $R$.

Thus, to follow the previously defined trajectory $\Gamma$ and estimate the grip conditions $\beta_{F}$ and $\beta_{R}$, state variables of the robot are defined as follows:

$\diamond s$, the robot curvilinear abscissa. It is the curvilinear distance along $\Gamma$ of point $M$, the point on $\Gamma$ that is the closest to $R$. The curvature of $\Gamma$ at point $M$ is denoted $c(s)$,

$\diamond y$, the robot tracking error or lateral deviation. It is the algebraic distance between $R$ and $M$,

$\diamond X$, the abscissa value of the center of the robot rear axle $R$ in the absolute frame,

$\diamond Y$, the ordinate value of the center of the robot rear axle $R$ in the absolute frame,

$\diamond \tilde{\theta}$, the robot angular error or angular deviation. It is the angle between the absolute robot heading, denoted $\theta$ and the orientation of the tangent to the trajectory at point $M$, denoted $\theta_{\text {tan }}$.

In this paper, additional notations will be added in the next sections to define the desired offsets applied to the robot moves when tracking. Thus, in the following, $y_{d}$ denotes the desired lateral deviation of the robot w.r.t. the trajectory while $\theta_{d}$ denotes the desired absolute orientation of the robot in the absolute frame.

\section{B. Motion model in the absolute frame}

According to classical kinematic analyses, such as those presented in [14], derivatives of the robot kinematic state variables (1) in the absolute frame can be written as follows:

$$
\left\{\begin{array}{rl}
\dot{X} & =v_{R} \cos \left(\theta+\delta_{R}+\beta_{R}\right) \\
\dot{Y} & =v_{R} \sin \left(\theta+\delta_{R}+\beta_{R}\right) \\
\dot{\theta} & =\frac{v_{F} \sin \left(\delta_{F}+\beta_{F}\right)-v_{R} \sin \left(\delta_{R}+\beta_{R}\right)}{L}
\end{array} .\right.
$$

The derivative of the state variable $\dot{\theta}$ is highly recommended in its given form which has no singularity and allows the continuity of the algorithms. This formula is used both in Secs. III and IV to define the evolution model of the proposed observer and the second part of the control algorithm. 


\section{Motion equations with respect to a reference trajectory}

According to classical kinematic analyses, such as those presented in [9], derivatives of the robot kinematic state variables (2) w.r.t. the reference trajectory can be written as follows:

$$
\left\{\begin{aligned}
\dot{s}= & v_{R} \frac{\cos \left(\tilde{\theta}+\delta_{R}+\beta_{R}\right)}{1-c(s) y} \\
\dot{y}= & v_{R} \sin \left(\tilde{\theta}+\delta_{R}+\beta_{R}\right) \\
\dot{\tilde{\theta}}= & \frac{v_{F} \sin \left(\delta_{F}+\beta_{F}\right)-v_{R} \sin \left(\delta_{R}+\beta_{R}\right)}{L} \\
& -v_{R} \frac{c(s) \cos \left(\tilde{\theta}+\delta_{R}+\beta_{R}\right)}{1-c(s) y}
\end{aligned}\right.
$$

This model is defined if the $1-c(s) y \neq 0$ condition is true. This means that the center of curvature $A$ should not be superimposed with the center of the rear axle $R$. However, if the robot is properly initialized, such a case is never reached in practice.

\section{ESTIMATION OF SIDESLIP ANGLES}

To ensure a suitable convergence of the tracking and angular errors along the trajectory when facing poor grip conditions, it is compulsory to know accurately the values of the sideslip angles. Because there is no direct perception system allowing to measure such variables, it has been shown in [10] that an observer may be built to estimate the two sideslip angles with a satisfying accuracy. To achieve this estimation, the robot must be equipped with on-board sensors that permit to measure the position, the orientation and the velocity of the robot at a given point, here $R$. Moreover, according to the model (1), defined above, the velocity of the robot at $F$, has to be measured.

\section{A. Observer state}

In this paper, the proposed observer in [10] built for a two-wheel steering mobile robot is extended to a four-wheel steering one, according to the equations of the model (1). Because this approach is a version close to [10], only a short presentation is proposed in this section.

Hereafter, $\xi$ should be consider as an effectively measured variable, while $\hat{\xi}$ should be considered as an observed or estimated variable. $\tilde{\xi}$ is defined as the observation error $\tilde{\xi}=\xi-\hat{\xi}$.

To achieve the indirect estimation of the sideslip angles, let us consider the state space $\xi$ defined as follows:

$$
\xi=\left[\begin{array}{c}
\xi_{p o s} \\
\xi_{\beta_{i}}
\end{array}\right] \text {, }
$$

where $\xi$ is split into two sub-states:

$\diamond \xi_{p o s}=\left[\begin{array}{lll}X & Y & \theta\end{array}\right]^{T}$, which constitutes the pose (position and orientation) of the robot in the absolute frame,

$\diamond \xi_{\beta_{i}}=\left[\begin{array}{ll}\beta_{F} & \beta_{R}\end{array}\right]^{T}$, which is composed of the sideslip angles, to be estimated.
Its evolution model is based on (1), and may be written as follows:

$$
\dot{\xi}=\left[\begin{array}{c}
\dot{\xi}_{p o s} \\
\dot{\xi}_{\beta_{i}}
\end{array}\right]=\left[\begin{array}{c}
f\left(\xi_{p o s}, \xi_{\beta_{i}}, v_{F}, v_{R}, \delta_{F}, \delta_{R}\right) \\
0_{2 \times 1}
\end{array}\right],
$$

where $f\left(\xi_{p o s}, \xi_{\beta_{i}}, v_{F}, v_{R}, \delta_{F}, \delta_{R}\right)$ is directly deduced from the three equations of model (1).

\section{B. Observer equations}

Equations for the observer are as follows:

$$
\dot{\hat{\xi}}=\left[\begin{array}{c}
\dot{\hat{\xi}}_{\text {pos }} \\
\dot{\hat{\xi}}_{\beta_{i}}
\end{array}\right]=\left[\begin{array}{c}
f\left(\xi_{\text {pos }}, \hat{\xi}_{\beta_{i}}, v_{F}, v_{R}, \delta_{F}, \delta_{R}\right)+\ldots \\
\ldots \alpha_{\text {pos }}\left(\tilde{\xi}_{\text {pos }}\right) \\
\alpha_{\beta_{i}}\left(\tilde{\xi}_{\text {pos }}\right)
\end{array}\right] \text {, }
$$

where $\alpha_{\text {pos }}\left(\tilde{\xi}_{p o s}\right)$ and $\alpha_{\beta_{i}}\left(\tilde{\xi}_{p o s}\right)$ are functions of the observation error attached to the pose part of the state $\xi$, and defined as follows:

$\left\{\begin{array}{l}\alpha_{\text {pos }}\left(\tilde{\xi}_{\text {pos }}\right)=K_{\text {pos }} \tilde{\xi}_{\text {pos }} \\ \alpha_{\beta_{i}}\left(\tilde{\xi}_{\text {pos }}\right)=K_{\beta}\left[\frac{\partial f}{\partial \xi_{\beta_{i}}}\left(\xi_{\text {pos }}, \hat{\xi}_{\beta_{i}}, v_{F}, v_{R}, \delta_{F}, \delta_{R}\right)\right]^{T} \tilde{\xi}_{\text {pos }}\end{array}\right.$,

where:

$\diamond K_{\text {pos }}$ is $3 \times 3$ positive diagonal matrice,

$\diamond K_{\beta}$ is a positive scalar.

As shown in [10] and extended to a four-wheel steering mobile robot, the observer defined by (5) and (6) allows the convergence of the whole observed state $\hat{\xi}$ to the actual one $\xi$. As a consequence, the values for the sideslip angles, $\hat{\beta}_{F}$ and $\hat{\beta}_{R}$ are obtained and can feed a control law appropriately in order to follow a path while keeping the same absolute orientation.

\section{CONTROL ALGORITHMS}

\section{A. Proposed strategy}

Previous approaches about parallel steering assume that the front and the rear steering are equal $\left(\delta_{F}=\delta_{R}\right)$ such as in [17]. However, if the grip conditions are not sufficient the vehicle will skid and deviate from the desired path.

Such a control strategy for each steering angle is not sufficient to ensure a suitable parallel steering. Indeed, even if the grip conditions are taken into account the robot will skid because its number of mobility degree is lower than the number of mobility required to ensure the tracking and the absolute orientation. In this paper, the strategy proposes to add a degree of mobility by independently controlling the rear and the front angles. The rear steering will ensure the convergence of the robot to the desired trajectory while the front steering will ensure in addition the convergence of the robot orientation to the desired one. This additional mobility 
will allow to correct the absolute orientation preventing the skid while following the path. Following subsections detail the backstepping strategy for each axle to reach this objective.

\section{B. Control algorithm for the rear steering angles}

The objective of this first step is to find a mathematical expression for $\delta_{R}$ that ensures the convergence of the rear lateral deviation $y$ to a desired lateral deviation $y_{d}$.

It has been chosen to set a convergence distance instead of a settling time to design a control strategy independent from time and the speed of the robot for the convergence of the lateral deviation. To obtain this kind of convergence it is compulsory to use derivatives of the state vector w.r.t. the curvilinear abscissa. Hereafter, $x^{\prime}$ should be consider as the derivative of $x$ w.r.t. the curvilinear abscissa $s$, such that $x^{\prime}=\frac{\mathrm{d} x}{\mathrm{~d} s}$.

Then from the product of the first equation of the model (2) with the inverse of the second one, the derivative of $y$ w.r.t. the curvilinear abscissa, denoted $y^{\prime}$, can be written as follows:

$$
y^{\prime}=\tan \left(\tilde{\theta}+\delta_{R}+\hat{\beta}_{R}\right)(1-c(s) y) .
$$

By denoting the error on the lateral deviation such that $e_{y}=y-y_{d}$, a way to ensure the convergence of this error to zero is to use a differential equation such that:

$$
e_{y}^{\prime}=y^{\prime}-y_{d}^{\prime}=k_{y} e_{y}
$$

with $k_{y}$ a negative scalar defining the convergence distance for the exponential convergence of $y$ to $y_{d}$ imposed by (8).

By injecting in this condition the expression of $y^{\prime}$ introduced in (7), one can then reformulate the previous conditions and obtain the following equation for each rear steering angle $\delta_{R}$ :

$$
\delta_{R}=\arctan \left(\frac{k_{y}\left(y-y_{d}\right)+y_{d}^{\prime}}{1-c(s) y}\right)-\tilde{\theta}-\hat{\beta}_{R} .
$$

This expression allows to ensure the differential Eq. (8) on lateral deviation $y$, implying its convergence to $y_{d}$. Limits for this expression are similar as those defined for expression (2).

\section{Control algorithm for the front steering angles}

The objective of this second step is to find a mathematical expression for $\delta_{F}$ that ensures both the convergence of the lateral deviation of the robot $y$ towards its desired value $y_{d}$ and the convergence of the absolute orientation of the robot $\theta$ towards its desired value $\theta_{d}$.

By denoting the error on the absolute angular deviation such that $e_{\theta}=\theta-\theta_{d}$, a way to ensure the convergence of this error to zero is to use a differential equation such that:

$$
\dot{e}_{\theta}=\dot{\theta}-\dot{\theta}_{d}=k_{\theta} e_{\theta}
$$

with $k_{\theta}$ a negative scalar defining the settling time for the exponential convergence of $\theta$ to $\theta_{d}$ imposed by (10).

By injecting in this condition the expression of $\dot{\theta}$ introduced in (1), one can then reformulate the previous conditions and obtain the following equation for each front steering angle $\delta_{F}$ :

$$
\delta_{F}=\arcsin \left(\frac{L\left(k_{\theta}\left(\theta-\theta_{d}\right)+\dot{\theta}_{d}\right)+v_{R} \sin \left(\delta_{R}+\hat{\beta}_{R}\right)}{v_{F}}\right)-\hat{\beta}_{F}
$$

After injecting in (11) the expression for $\delta_{R}(9)$ previously introduced, one can finally obtain the following equation for each front steering angle $\delta_{F}$ :

$$
\begin{aligned}
\delta_{F}= & \arcsin \left(\frac{L\left(k_{\theta}\left(\theta-\theta_{d}\right)+\dot{\theta}_{d}\right)}{v_{F}}\right. \\
& \left.+\frac{v_{R} \sin \left(\arctan \left(\frac{k_{y}\left(y-y_{d}\right)+y_{d}^{\prime}}{1-c(s) y}\right)-\tilde{\theta}\right)}{v_{F}}\right)-\hat{\beta}_{F},
\end{aligned}
$$

provided that $v_{F} \neq 0$ and limits similar as those defined for expression (9). In practice, the velocity is always positive when path tracking and consequently non null. This expression allows then to ensure the differential Eq. (8) on lateral deviation $y$, implying its convergence to $y_{d}$. In addition, this expression also ensures the convergence of the absolute orientation $\theta$ to its desired value $\theta_{d}$.

Finally, by using expressions (9) and (12) for the rear and the front angles coming from the proposed backstepping strategy and by settling the desired lateral deviation and orientation, $y_{d}$ and $\theta_{d}$, the objective introduced by this paper (i.e. control the absolute orientation of the robot and its lateral position w.r.t. the trajectory) is achieved.

\section{Predictive curvature servoing}

To anticipate trajectory overshoots due to the actuator settling time, a predictive curvature servoing has been implemented. Only a few details about these control algorithms are given in this section. Nevertheless, it follows the same methodology as the one detailed in [3].

Knowing the evolution of the reference path (i.e. computed or previously learned), the reference path curvature can be anticipated. To achieve this, the future curvature of the reference path is computed w.r.t. a prediction time denoted $t_{\text {pred }}$. This future curvature is then injected in the steering laws to ensure the equality between the trajectory and vehicle curvature.

\section{SIMULATION RESULTS}

Making use of the proposed strategy based on the observer, detailed in Sec. III, and the control laws, detailed above, 
algorithms have been tested on a MATLAB/ADAMS cosimulator with the simulated vehicle depicted in Fig. 2. This vehicle is four-wheel drive and has four independent steering wheels without steering range. Its features are described in Table I. These simulations are realistic w.r.t. technologies met in current industries. Indeed, the vehicle is equipped with a simulated IMU and a simulated RTK-GPS. The sampling rate for these trials has been set at $10 \mathrm{~Hz}$ as in common real-time mobile robotic applications.

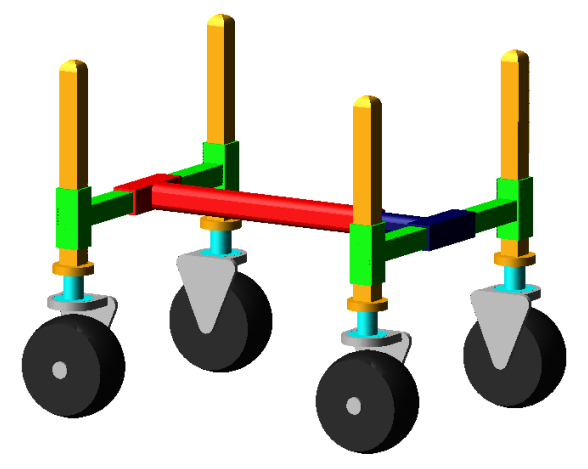

Fig. 2: 4WS mobile robot simulator.

TABLE I: Vehicle features.

\begin{tabular}{|c||c|}
\hline Weight & $900 \mathrm{~kg}$ \\
\hline Wheelbase (L) & $2 \mathrm{~m}$ \\
\hline Trackbase & $1.2 \mathrm{~m}$ \\
\hline Steering angle response time & $0.24 \mathrm{~s}$ \\
\hline
\end{tabular}

For the following simulations, the values of the gains have been chosen as follows: $k_{y}=-0.55$ and $k_{\theta}=-0.8$. The robot speed has been set at $2 \mathrm{~m} / \mathrm{s}$ according to the work speeds usually used in many agricultural contexts.

Successive robot positions (seen from above: ::: have been added in the following tracking figures to illustrate the motion of the robot when tracking.

\section{A. Straight line tracking}

The objective of this trial is the verification of the proposed control strategy for parallel steering during straight line tracking when the robot is given different desired lateral deviations and absolute orientations. For this we have imposed to the vehicle the reference path depicted in black in Fig. 3 and Fig. 4.

As illustrated in Fig. 3, the vehicle is first located at an initial distance to the trajectory $y_{\text {init }}$ equal to $0.5 \mathrm{~m}$ (1)). At the beginning ( $t=0 \mathrm{~s}$ ), the robot is given a desired lateral deviation equal to zero $\left(y_{d}=0 \mathrm{~m}\right)$ and a desired absolute orientation equal to zero $\left(\theta_{d}=0\right.$ degrees) (2)). Then, at $t=10 \mathrm{~s}$, the lateral deviation is set at $0.3 \mathrm{~m}$ (3) and then set, at $t=15 \mathrm{~s}$, at zero again $\left(y_{d}=0 \mathrm{~m}\right)$ (4)). At $t=20 \mathrm{~s}$, the robot is given a desired absolute orientation equal to 25 degrees $\approx 0.436$ radians (5). Then, the desired lateral deviation is set at several values while keeping this absolute orientation. Then, at $t=30 \mathrm{~s}$, the desired lateral deviation is set at $0.4 \mathrm{~m}$ (6), then, at $t=40 \mathrm{~s}$, the desired lateral deviation is set at $-0.2 \mathrm{~m}$ (7) and finally, at $t=45 \mathrm{~s}$, the desired lateral deviation is set to zero $\left(y_{d}=0 \mathrm{~m}\right)$ (8).

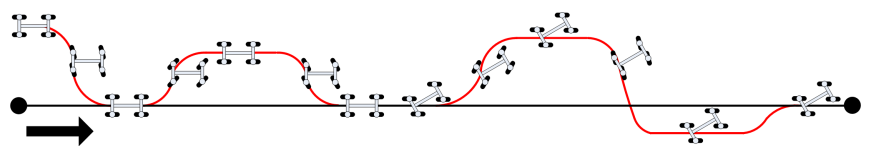

Fig. 3: Reference path (black), expected path (red) and successive robot positions $(\because:)$ ).

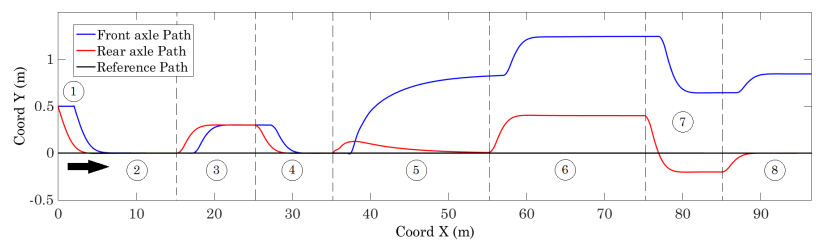

Fig. 4: Reference path (black) and tracking (blue and red).

The results for the lateral deviation $y$ and the absolute orientation $\theta$ of the robot are depicted in Figs. 5 and 6.

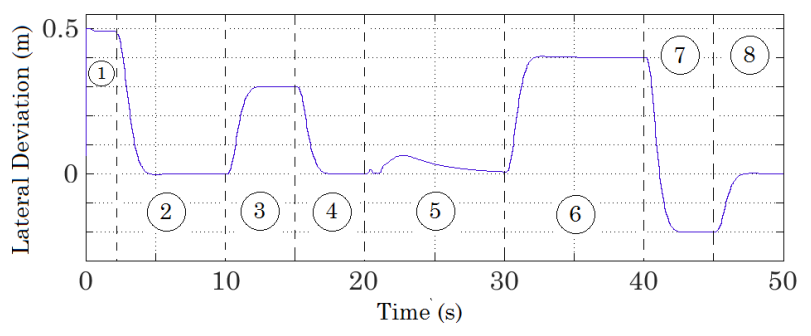

Fig. 5: Lateral deviation results.

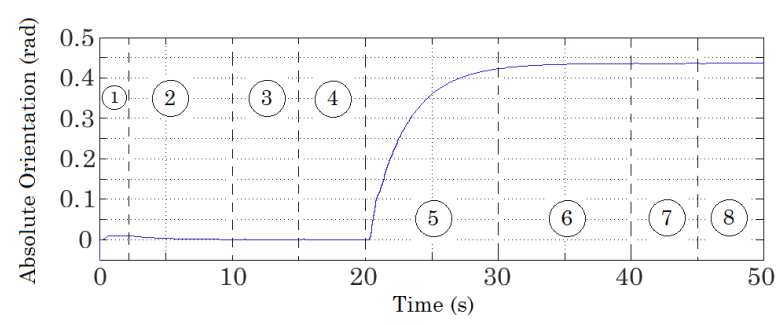

Fig. 6: Absolute orientation results.

It is apparent in Fig. 5 that the proposed parallel steering strategy ensures the convergence of the robot towards different desired lateral deviations during straight line tracking whatever the absolute orientation of the robot. Moreover, as depicted in Fig. 6 the proposed strategy ensures the convergence of the absolute orientation of the robot to different desired orientations while ensuring the convergence of the lateral deviation. 


\section{B. Curve tracking}

The objective of this trial is the verification of the proposed control strategy for parallel steering during curve tracking when the robot is given different desired lateral deviations and absolute orientations. For this we have imposed to the vehicle the trajectory depicted in black in Fig. 7.

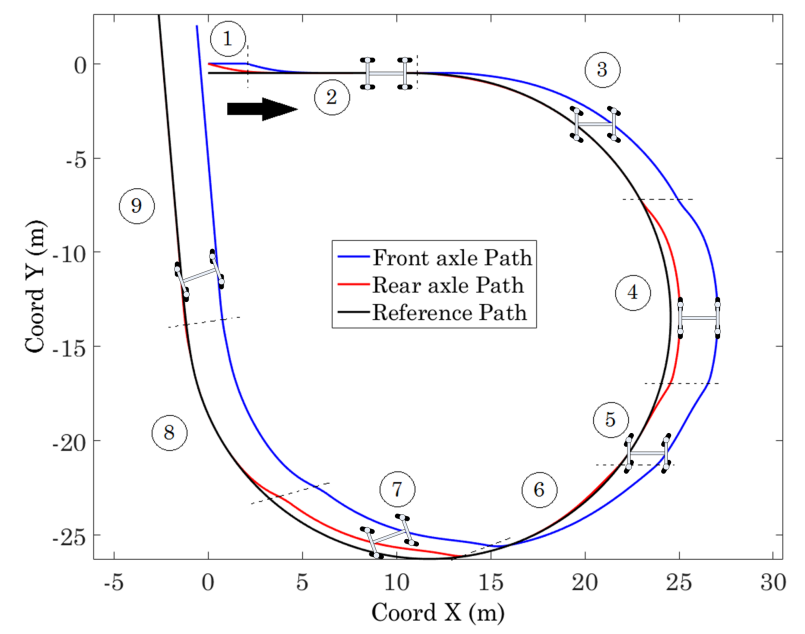

Fig. 7: Reference path (black), tracking (blue and red) and successive robot positions $(\mathbb{2}:)$.

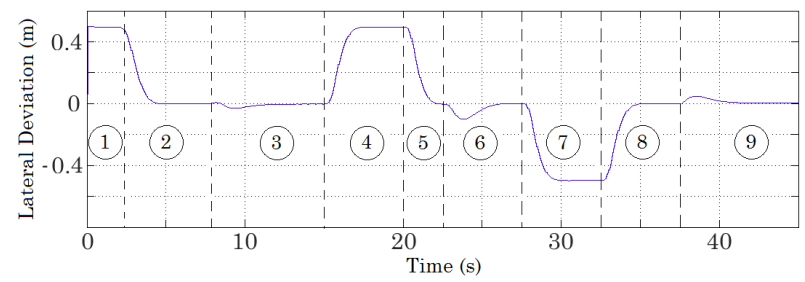

Fig. 8: Lateral deviation results.

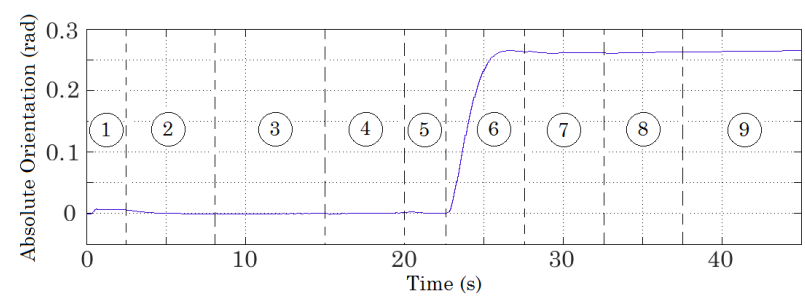

Fig. 9: Absolute orientation results.

As illustrated in Fig. 7, the vehicle is first located at an initial distance to the trajectory $y_{\text {init }}$ equal to $0.5 \mathrm{~m}$ (1). At the beginning $(t=0 \mathrm{~s})$, the robot is given a desired lateral deviation equal to zero $\left(y_{d}=0 \mathrm{~m}\right)$ and a desired absolute orientation equal to zero $\left(\theta_{d}=0\right.$ degrees) (2)). After a short straight line, the robot is asked to follow a curved trajectory (3). Then, while tracking this curve, the desired lateral deviation is firstly set to $0.5 \mathrm{~m}$ at $t=15 \mathrm{~s}$ (4)), then to $0 \mathrm{~m}$ at $t=20 \mathrm{~s}$ (5). At $t=22.5 \mathrm{~s}$ the desired absolute orientation is set to 15 degrees $\approx 0.262$ radians (6)). Then, the desired lateral deviation is set to $-0.5 \mathrm{~m}$ at $t=27.5 \mathrm{~s}$ (7) and finally to zero at $t=32.5 \mathrm{~s}$ (8)). When the curve stops, the trajectory becomes again a straight line (9).

The results for the lateral deviation $y$ and the absolute orientation $\theta$ of the robot are depicted in Figs. 8 and 9.

It is apparent in Fig. 8 that the proposed parallel steering strategy ensures the convergence of the robot towards different desired lateral deviations during curve tracking whatever the absolute orientation of the robot. Moreover, as depicted in Fig. 9 the proposed strategy ensures the convergence of the absolute orientation of the robot to different desired orientations while ensuring the convergence of the lateral deviation.

\section{Manoeuvres applied to agricultural robotics}

From previous simulations, one can see that the proposed parallel steering strategy is suitable for achieving accurate path tracking in off-road conditions. It is then possible to imagine several scenarios with this kind of control in different agricultural contexts where manoeuvres are tricky because of the lack of space. It is the case for instance when facing narrow parking places or doing U-turns between vine rows. We have chosen to illustrate these situations with the relevant simulations depicted in Figs. 10 and 11 that show the achievements and the accuracy of using the proposed parallel steering strategy in such situations.

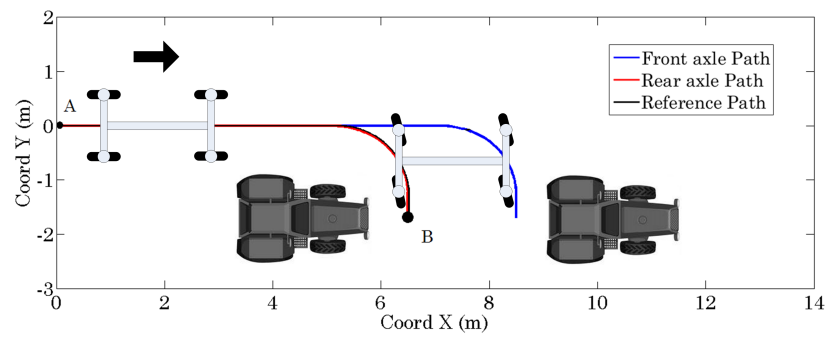

Fig. 10: Reference path (black), tracking (blue and red) and successive robot positions ( $: \mathbf{a}$ ) when parking.

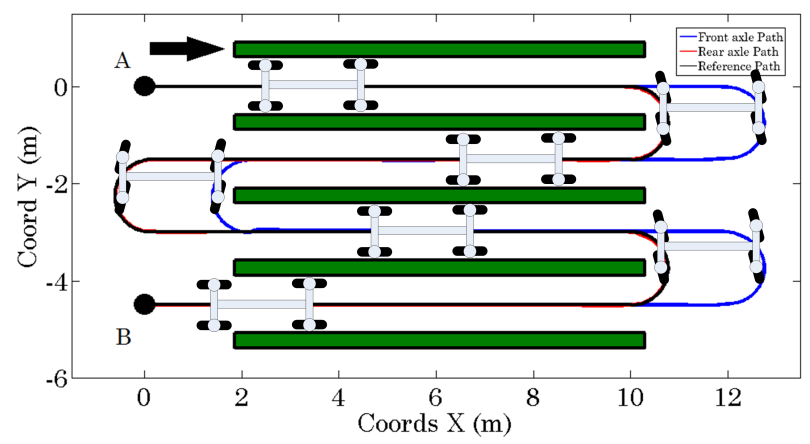

Fig. 11: Reference path (black), tracking (blue and red) and successive robot positions $(: \mathbb{Z})$ between vine rows (green). 


\section{CONCLUSION}

This paper proposes a parallel steering strategy dedicated to a four-wheel steering mobile robot in off-road contexts. Because of poor grip conditions and imposing an equal angle to the front and the rear axles, previous parallel steering approaches cannot ensure an accurate tracking because of the skid and the deviation from the desired path. Here, the grip conditions are considered by using an observer based on the extended kinematic model of the robot. This observer, derived from previous work, allows suitable estimations for the sideslip angles of a four-wheel steering mobile robot. These estimations then feed the proposed control laws based on a backstepping strategy ensuring both the convergence of the robot towards a desired lateral deviation and a desired absolute orientation. This new point of view allows to avoid possible skids while guaranteeing the accuracy of the tracking. As shown in the simulation section, the combination of the observer with the proposed control laws leads to high accurate path tracking and can be used for tricky manoeuvres in agricultural contexts for instance. Future work will be focused on the design and the implementation of an observer dedicated to each wheel of the robot to estimate more precisely sideslip angles to further increase the accuracy of the path tracking.

\section{ACKNOWLEDGMENT}

This work has received the support of the French National Research Agency under the grant number ANR-14-CE270004 attributed to AdAP2E project and has been sponsored by the IMobS3 Laboratory of Excellence under the grant number ANR-10-LABX-16-01.

\section{REFERENCES}

[1] G. Bayar, M. Bergerman and A. B Koku (2016). Improving the trajectory tracking performance of autonomous orchard vehicles using wheel slip compensation. Biosystems Engineering, vol. 146, pp. 149164.

[2] M. Bergerman, J. Billingsley, J. Reid and E. van Hentenl (2016). Robotics in Agriculture and Forestry. In : Springer Handbook of Robotics. Springer International Publishing, p. 1463-1492.

[3] C. Cariou, R. Lenain, B. Thuilot and M. Berducat (2009). Automatic guidance of a four-wheel steering mobile robot for accurate field operations. Journal of Field Robotics, vol. 26, no 6-7, pp. 504-518.

[4] R. Fierro and F L. Lewis (1995). Control of a nonholonomic mobile robot: backstepping kinematics into dynamics. In : Decision and Control, Proceedings of the 34th IEEE Conference on. IEEE, p. 38053810.

[5] W. Grey Walter (1950). An imitation of life. Scientific American, vol. 182, no 5, pp. 42-45.

[6] L. Grimstad, C. Pham, H. Phan and P. From (2015). On the design of a low-cost, light-weight, and highly versatile agricultural robot. IEEE International Workshop on Advanced Robotics and its Social Impacts (ARSO), pp. 1-6.

[7] L. Haibo, D. Shuliang, L. Zunmin and Y. Chuijie (2015). Study and experiment on a wheat precision seeding robot. Journal of Robotics, pp. 12.

[8] K. Kozlowski and D. Pazderski (2004). Modeling and control of a 4-wheel skid-steering mobile robot. Int. J. Appl. Math. Comput. Sci, vol. 14, no 4, p. 477-496.

[9] R. Lenain, B. Thuilot, C. Cariou and P. Martinet (2006). High accuracy path tracking for vehicles in presence of sliding: Application to farm vehicle automatic guidance for agricultural tasks. Autonomous Robots, vol. 21 , no. 1 , pp. 79-97.
[10] R. Lenain, M. Deremetz, J-B. Braconnier, B. Thuilot and V. Rousseau (2017). Robust sideslip angles observer for accurate off-road path tracking control. Advanced Robotics, pp. 1-15.

[11] F. Marmoiton and M. Slade (2016). Toward Smart Autonomous Cars. Intelligent Transportation Systems: From Good Practices to Standards, pp. 84-109.

[12] P. Moubarak and P. Ben-Tzvi (2012). Modular and reconfigurable mobile robotics. Robotics and Autonomous Systems, vol. 60, no 12, p. $1648-1663$.

[13] C. Samson (1995). Control of chained systems. Application to path following and time-varying point stabilization of mobile robots. IEEE Transactions on Automatic Control, vol. 40, no. 1, pp. 64-77.

[14] C. Samson, P. Morin and R. Lenain (2016). Modeling and control of wheeled mobile robots. In : Springer Handbook of Robotics. Springer International Publishing, p. 1235-1266.

[15] P. Sapaty (2015). Military robotics: Latest trends and spatial grasp solutions. International Journal of Advanced Research in Artificial Intelligence, vol. 4, no 4, p. 9-18.

[16] B. Thuilot, C. Cariou, P. Martinet and M. Berducat (2002). Automatic guidance of a farm tractor relying on a single CP-DGPS. Autonomous robots, vol. 13, no 1, pp. 53-71.

[17] D. Wang and F. Qi (2001). Trajectory planning for a four-wheelsteering vehicle. IEEE International Conference on Robotics and Automation (ICRA), vol 4, pp. 3320-3325. 